\title{
Évaluation des enjeux sociaux lors de l'implantation d'une coopérative d'épargne et de crédit au Mali
}

\section{Claude Rossi}

\author{
(2) OpenEdition \\ Journals \\ Édition électronique \\ URL : http://journals.openedition.org/apad/2523 \\ DOI : 10.4000/apad.2523 \\ ISSN : 1950-6929 \\ Éditeur \\ LIT Verlag \\ Édition imprimée \\ Date de publication : 1 décembre 1993 \\ Référence électronique \\ Claude Rossi, «Évaluation des enjeux sociaux lors de l'implantation d'une coopérative d'épargne et de \\ crédit au Mali », Bulletin de l'APAD [En ligne], 6 | 1993, mis en ligne le 10 mars 2008, consulté le 08 \\ septembre 2020. URL : http://journals.openedition.org/apad/2523 ; DOI : https://doi.org/10.4000/ \\ apad. 2523
}

Ce document a été généré automatiquement le 8 septembre 2020

Bulletin de I'APAD 


\title{
Évaluation des enjeux sociaux lors de l'implantation d'une coopérative d'épargne et de crédit au Mali
}

\author{
Claude Rossi
}

1 Cet article traite du changement social introduit par l'implantation d'une coopérative d'épargne et de crédit (CEC) dans un village minyanka de la région de Ségou au Mali : M'Pétiona. Sur la base des connaissances accumulées, j'ai présumé que ce projet constituerait un enjeu qui actionnerait les groupes sociaux ayant des intérêts divers dans le village. J'ai donc proposé une analyse de l'impact social de ce projet à travers les réactions du pouvoir local et celles des différents groupes sociaux villageois.

Pouvoir local et changement social dans les communautés villageoises africaines

2 L'étude du changement social peut se faire de différentes façons. Après avoir constaté les limites propres à certains courants de pensée, en particulier le fonctionnalisme et le structuralisme, nous avons retenu les possibilités qu'offrent l'approche dynamiste et le courant marxiste.

3 À la suite de plusieurs auteurs (Balandier, 1971, 1984; Terray, 1969; Copans 1975; Meillassoux, 1977 ; Amselle, 1985, etc. en ce qui concerne l'Afrique mais aussi Ledrut, 1979; Bariteau et al. 1983 et Vergès, 1983 à propos du pouvoir local dans d'autres contextes), nous avons tenté de tirer profit d'une approche qui cherche à privilégier la dynamique sociale, approche qui s'appuie sur des concepts opérationnels et qui a déjà permis de faire ressortir les effets sur les entités locales des changements venant de l'extérieur. Nous avons retenu de ces études l'importance de la dimension historique pour comprendre comment les changements se sont développés localement et quels sont les vecteurs de ces changements.

Pouvoirs villageois et modèles de développement

4 De manière générale, comme le mentionnent plusieurs analystes (Billaz, 1981, Haubert, 1985, Amselle, 1985, etc.) les effets des projets se traduisent par un renforcement de la différenciation sociale entre, d'une part, la paysannerie et la classe dominante et, d'autre part, à l'intérieur des communautés, entre les divers groupes sociaux. 
On constate une diminution de la taille des unités de production suite à la division des grandes communautés familiales et à la volonté d'autonomie des jeunes. Cela se traduit souvent par un accroissement du travail demandé aux femmes comme le soulignent plusieurs recherches (entre autres, Dumouchel et Thède, 1986 et Guillermou, 1988, etc.).

6 Les opérations de développement, tout en visant une amélioration de la situation socio-économique des ruraux, risquent en fait dans bien des cas de contribuer au renforcement des inégalités :

- au niveau interne, ces pratiques accentuent l'exploitation des femmes, les conflits entre ânés et cadets et les privilèges des groupes dominants ;

- au niveau externe, les projets permettent un contrôle accru et une ponction croissante des classes dominantes sur la production paysanne, favorisant ainsi la différenciation sociale.

Conditions pour une étude du pouvoir local dans une communauté villageoise

7 Il ressort de l'examen des changements sociaux survenus dans les communautés villageoises africaines que l'analyse de ceux-ci ne peut se faire sans tenir compte de ce qui a cours aux niveaux interne et externe à l'unité d'analyse.

8 Au niveau interne, il importe d'identifier l'organisation socio-économique locale et les groupes sociaux qui la composent. Ces groupes peuvent être caractérisés par des différences d'âge, de sexe, par leur appartenance ethnique, par leur orientation religieuse et d'autres variables spécifiques selon les cas. Ces informations doivent être introduites dans l'analyse des organisations locales, dans celle de l'histoire du milieu, dans l'analyse des rapports entre les groupes du milieu et dans l'analyse des rapports entre ces derniers et les classes dominantes.

9 Au niveau externe, il faut préciser quels sont les intervenants qui agissent et qui ont un intérêt dans la communauté en spécifiant leurs liens avec les groupes sociaux locaux ou avec les organisations du milieu. Ces agents des classes dominantes peuvent être des représentants des organismes publics, para-publics ou privés, des commerçants, des agences de développement, etc.

Questionnement et objectif de la recherche

10 À la lumière des travaux effectués sur des cas analogues, j'ai présumé que, contrairement aux objectifs du projet de CEC, ce serait certains groupes, en particulier ceux associés aux classes dominantes par leurs activités économique et politique et par leur orientation religieuse, qui seraient favorisés. Compte tenu de ces informations, je pensais que l'enjeu se situerait entre les groupes détenant un pouvoir traditionnel en transformation, les aînés, et ceux détenant un pouvoir moderne appuyé par la classe dominante, soit les jeunes et les adultes alors que les femmes, contrairement aux objectifs du projet, seraient marginalisées par rapport à la CEC.

11 C'est l'analyse du champ politique local qui devait me permettre d'identifier les groupes et leur position face à cette intervention. Pour cela, j'ai examiné le processus d'implantation de la CEC dans le village et $j$ 'en ai fait une analyse sous l'angle des agents actifs en les situant par rapport aux différents groupes identifiés, aux organisations villageoises et à la structure du pouvoir local.

12 Rappelons que ce projet émane de la Société de Développement International Desjardins (SDID) qui, pour faciliter ses activités d'implantation, a formé un comité de concertation composé d'organisations maliennes actives dans le village. Il faut aussi mentionner que le type de coopérative proposé, le modèle Desjardins, est indépendant 
des organisations économiques locales et doit fonctionner de manière autonome en impliquant toutes les catégories sociales du milieu. Il existe par contre d'autres modèles (européens) de caisse d'épargne en cours d'implantation dans la région qui s'appuient sur les associations villageoises pour s'implanter dans le milieu.

Méthodologie

Par une revue de littérature sur la problématique, j'ai pu accéder à une connaissance générale de la situation socio-économique, de l'histoire et des transformations en cours dans les communautés rurales africaines, maliennes et, en particulier, dans la région minyanka du Mali où se trouve le village de M'Pétiona. J'ai précisé mon approche théorique du pouvoir local en retenant les approches dynamiste et marxiste et en les adaptant à la réalité rurale africaine. Pour les fins de cette analyse j'utilise les concepts de "champ politique local", "groupes sociaux localisés" et "classe dominante". Nous retenons aussi les concepts de "pouvoir endogène" et "pouvoir exogène" pour caractériser le pouvoir local en regard des différents groupes sociaux.

Pour appréhender les groupes sociaux localisés je me sers des catégories d'âge (aîné/ adulte/jeune), de sexe (femme/homme), de l'origine ethnique (minyanka/peul), clanique (pour les forgerons) et de l'orientation religieuse (animiste/musulman).

La méthode de collecte des données et le choix des indicateurs découlent de l'approche théorique. Je pense, comme Labatut (1989), qu'une méthode anthropologique qui intègre la dynamique locale dans le contexte global est mieux adaptée pour aborder ce type de problématique en Afrique. Par ailleurs, sur le terrain, les méthodes qualitatives utilisées en anthropologie pour les analyses micro-sociales, soit l'observation participante, le recours à des informateurs-clé, des entrevues semi-dirigées et des discussions informelles ont été privilégiées. Elles permettent une saisie plus globale de la réalité locale.

Cette recherche étant réalisée en collaboration avec la SDID, j'ai eu plusieurs rencontres avec le responsable des CEC au Mali au cours de l'année 1990 pour préparer notre collecte des données. Le séjour au Mali, soit à Ségou et M'Pétiona, s'effectua de septembre à décembre 1990. A Ségou où résidaient les intervenants du projet, j'ai pris connaissance des études de la SDID (1990) concernant le village de M'Pétiona.

Lors de mon arrivée à M'Pétiona, mon premier travail a consisté à sillonner le village, à identifier les nombreux quartiers, à discuter avec les villageois et à connaître les principaux leaders. Par la suite, deux entrevues semi-dirigées ont été réalisées avec des intervenants externes connaissant bien le village. Ces entretiens m'ont permis de préciser le rôle des intervenants externes dans le village et dans le projet de CEC.

Dans les entrevues avec les villageois, j'ai cherché à préciser le rôle et les caractéristiques sociales, politiques, économiques et idéologiques des différents groupes sociaux. J'ai tenté de situer, dans une perspective historique, les groupes potentiellement antagonistes en précisant quels sont ou quels ont été leurs rôles dans les organisations locales et quels sont leurs rapports avec les classes dominantes. Ceci a permis d'identifier les principaux leaders dans les organisations du village. Les informateurs appartenaient aux différents groupes sociaux du milieu.

19 Il m'a été difficile, en tant qu'homme étranger, d'interroger des femmes. J'ai tout de même réalisé trois entrevues, deux avec des jeunes femmes et une avec une accoucheuse traditionnelle (une aînée). Par ailleurs, j'ai discuté avec plusieurs villageoises. 
20 J'ai porté une attention particulière au processus d'implantation de la CEC pour cerner les personnes actives. L'observation et la participation aux réunions de celles-ci ont été utilisées à cette fin.

21 L'analyse des données a été divisée en trois parties que je présente dans les prochains paragraphes. La première débute par une description générale du Mali, de la région minyanka et des particularités socio-économiques et historiques du village. Dans la deuxième partie, ce sont les organisations politiques et économiques présentes dans le milieu qui retiendront mon attention pour situer la dynamique du pouvoir local. Après avoir décrit les activités d'implantation de la $\mathrm{CEC}$, dans la troisième partie, je ferai ressortir les caractéristiques des groupes sociaux localisés en regard de la CEC. La conclusion intégrera les changements en cours dans le village dans une perspective plus globale.

Le changement social dans un village minyanka du Mali

22 Au Mali, la classe dominante, issue de l'indépendance, assure toujours son pouvoir dans le pays en contrôlant la production agricole. Devant la crise financière du pays, les politiques économiques de cette classe dépendent en grande partie de financements extérieurs. Les organismes financiers ont imposé une libéralisation de l'économie qui a favorisé les entreprises et les commerçants privés.

Dans la région minyanka, la Compagnie Malienne de Développement du Textile (CMDT) et l'administration appuient la culture du coton qui procure des revenus substantiels à l'Etat et à cette compagnie. La commercialisation du coton est assurée par la CMDT. Les commerçants privés concentrent leurs activités sur la commercialisation du mil, sur celle de productions diverses et sur l'importation de biens de consommation.

Ces changements se traduisent chez les paysans de M'Pétiona par un abandon d'une partie des réserves traditionnelles de mil et par une diminution des grosses communautés familiales. Malgré la présence d'organisations externes, l'introduction d'activités de type capitaliste (production de coton) et l'islamisation croissante, l'organisation socio-économique reste communautaire et les aînés conservent un rôle politique, économique et idéologique dominant. La division des activités socio-économiques est encore marquée par le contrôle des aînés sur les cadets et sur les femmes.

Par ailleurs, le village de M'Pétiona a été divisé en deux entités administratives à la suite d'un malentendu entre les premiers occupants, plus musulmans, et des groupes récemment implantés, plus animistes. Il y a donc deux chefs. Un à M'Pétiona 1 (P1) et un à M'Pétiona 2 (P2). Pour ce qui est des groupes ethniques, le village est majoritairement minyanka avec cependant des quartiers peul et un quartier de forgerons.

26 Les caractéristiques des organisations formelles et traditionnelles, qui jouent un rôle économique et social pour plusieurs villageois et villageoises, nous indiquent la situation différenciée des hommes et des femmes par rapport à celles-ci.

Les hommes contrôlent l'organisation économique formelle, l'Association Villageoise (AV) commune aux deux M'Pétiona, qui soutient la production du coton et dans laquelle il n'y a pas de femmes. Ces dernières sont surtout actives dans les organisations traditionnelles ou informelles, les tons et les tontines, où l'on retrouve aussi des hommes. 
Ces organisations informelles se caractérisent, entre autres, par des pratiques d'épargne et de crédit associées à des groupes d'âge et de sexe. Ces pratiques ne favorisent pas a priori l'épargne monétaire dans une organisation formelle commune à tous les villageois comme le souhaite le projet de CEC.

Les différents groupes sociaux, ethniques, religieux, l'histoire de la division du village et l'organisation socio-économique illustrent la diversité existant localement et les divergences potentielles entre certains groupes.

Cette diversité s'enracine localement autour de quatre groupes principaux :

1) les hommes aînés, plus animistes à P1 et plus musulmans à P2, qui sont les leaders traditionnels ;

2) les jeunes hommes ;

3) les hommes adultes ;

Ces deux derniers groupes sont généralement musulmans et plus ouverts aux innovations et aux changements.

4) les femmes qui suivent l'appartenance religieuse du chef de famille et semblent marginalisées dans le pouvoir local, autant à P1 qu'à P2.

Les groupes sociaux localisés et le pouvoir local

La présentation de l'organisation politique et économique permet de préciser la dynamique du champ politique local de M'Pétiona à partir des quatre groupes sociaux localisés identifiés.

Les aînés demeurent des leaders traditionnels dans le village et conservent leur pouvoir dans les organisations formelles, celles en contact avec les classes dominantes maliennes.

Par contre, ce sont souvent les adultes qui établissent les contacts avec les intervenants externes comme l'administration, la CMDT, etc. Les aînés ne sont pas réfractaires aux changements mais ils veulent les contrôler. Ils gardent un pouvoir de décision à l'interne et travaillent avec les adultes pour les contacts avec l'extérieur. Ils nous disaient que "le monde ne va pas à l'envers et que pour aller dans le vestibule des jeunes il faut passer par le vestibule des vieux". Ils font ainsi référence à la position des habitations dans les quartiers et à leur contrôle sur les innovations introduites dans le village.

39 En prenant en considération l'appartenance religieuse, on remarque que les groupes les plus portés vers des activités économiques externes, les commerçants, certains membres de l'AV et des conseillers villageois, sont composés d'adultes musulmans, majoritairement de P2. On se souviendra que la séparation du village s'est articulée, entre autres, autour de clivages religieux. Les gens de P1 étaient animistes et ceux de P2 déjà plus musulmans. Actuellement, les aînés de P1 sont encore en grande partie animistes mais les jeunes et les adultes se disent plutôt musulmans. L'islam est en progression dans tout le village mais nettement plus présent à P2.

40 En tenant compte des variables ethniques, claniques et religieuses, on s'aperçoit que ce sont les Minyanka, surtout musulmans mais aussi animistes, la majorité de la population, qui contrôlent le pouvoir local. Mais les Peul et un forgeron sont représentés dans les organisations formelles et la plupart de ceux-ci sont musulmans.

41 Les deux autres groupes sociaux localisés, les femmes et les jeunes hommes, ont peu de place dans les principales organisations du village quelque soit leur appartenance 
ethnique, clanique ou religieuse. Les femmes sont surtout actives dans les organisations traditionnelles locales. Les jeunes hommes sont plus en contact avec l'extérieur et ils sont généralement musulmans. Ils sont près de l'AV et des commerçants et, même s'ils n'ont pas de pouvoir visible, ils introduisent des innovations dans le milieu. Ils ont une influence idéologique.

La classe dominante malienne intervient au village à travers différents organismes, la CMDT, le Centre d'Action Coopérative (CAC), le Centre de Formation des Animatrices Rurales (CFAR), l'administration et l'école. Mais c'est la CMDT qui a les principales activités au village. Cette compagnie appuie la culture du coton et forme des jeunes et des adultes pour cela. Les autres organisations externes viennent en quelque sorte compléter les activités de la CMDT. Le CAC contrôle l'AV, le CFAR forme des femmes, l'école forme des enfants et l'administration collecte les impôts lorsque les revenus du coton sont rentrés.

Pour contrôler ses intérêts dans le village, c'est-à-dire la production de rente qui permet de dégager le surplus, la classe dominante forme et contrôle les différents groupes sociaux villageois et s'associe aux hommes adultes pour promouvoir la culture du coton.

44 C'est le groupe qui partage avec celle-ci certains intérêts économiques et la même religion. Toutefois, à l'interne, les adultes continuent d'appuyer et de respecter le pouvoir des aînés.

Le champ politique local se caractérise donc par un contrôle des aînés sur l'organisation politique et socio-économique villageoise. L'organisation politique officielle est divisée entre les deux entités administratives avec à sa tête, à P1, des aînés animistes, et, à P2, des aînés musulmans. Ces deux catégories se regroupent dans l'AV où la majorité des membres, des aînés et des adultes des deux M'Pétiona, sont musulmans.

Les rapports avec les organisations externes sont établis par les adultes musulmans qui ont un rôle significatif dans les organisations villageoises et qui favorisent la réconciliation des deux villages dans l'AV comme le font d'ailleurs les organismes externes. Ceux-ci s'appuient sur le groupe qui, tout en étant ouvert aux innovations, détient un certain pouvoir politique, économique et idéologique dans le village : les hommes adultes musulmans.

Les nouveaux organismes comme l'AV se constituent à partir de ce groupe homogène qui présente moins d'antagonismes que les aînés. Ces derniers sont en effet toujours divisés par l'histoire du milieu et l'appartenance religieuse. Le pouvoir endogène est donc contrôlé par les aînés et le pouvoir exogène est introduit par les adultes et, dans une moindre mesure, par les jeunes.

Le pouvoir local et la coopérative d'épargne et de crédit

L'observation des activités d'implantation, des membres du conseil d'administration et des caissiers de la CEC nous indique que ce sont surtout les jeunes hommes des deux M'Pétiona qui sont actifs. Les autres groupes sociaux localisés sont peu présents dans les débuts de la CEC.

49 La stratégie de la SDID qui a consisté à former un comité de concertation composé d'organisations externes (CMDT, CAC et CFAR) encourage la participation des jeunes. C'est ce groupe homogène, celui des jeunes hommes musulmans, qui s'est activé autour de la CEC. Ce sont ces mêmes jeunes qui sont liés aux classes dominantes, qui ont des 
affinités religieuses avec celles-ci, ce sont eux qui favorisent la réconciliation des deux villages.

Par contre, les leaders traditionnels ne constituent pas un groupe homogène, certains à M'Pétiona 1 sont encore animistes, d'autres à M'Pétiona 2 sont musulmans. C'est un groupe encore divisé par l'histoire du village. La stratégie d'implantation du projet a eu pour effet d'écarter ce groupe qui détient pourtant un pouvoir endogène significatif. Les adultes eux aussi sont peu présents dans les activités de la Caisse et se situent comme les aînés.

Les femmes, elles aussi, ont été peu sensibilisées et sont peu actives dans les débuts de la Caisse. On peut donc supposer que cette stratégie ne favorisera pas, à court terme, une adhésion importante des villageois et des villageoises à la CEC.

Par ailleurs, cette intervention, en s'appuyant uniquement sur les jeunes hommes, risque d'accentuer la différenciation sociale en cours dans le milieu comme nous l'avons mentionné à la suite de plusieurs auteurs.

53 On voit donc les limites d'une stratégie qui vise à favoriser les intervenants externes dans les activités d'implantation. Les leaders locaux semblent mettre entre les mains des jeunes hommes une organisation dont ils connaissent peu les avantages et qui est appuyée et contrôlée de l'extérieur. Les jeunes, visiblement plus attirés vers la nouveauté, se retrouvent ainsi associés à ces organismes et à la CEC.

Le fait de favoriser la collaboration avec des organismes externes a eu pour conséquence d'impliquer les jeunes dans les activités du projet au détriment des autres groupes sociaux localisés et des organisations villageoises. Des collaborations significatives avec les membres de l'AV et avec des groupes de femmes auraient pu accélérer l'implantation et le membership de la CEC. organismes actifs dans le milieu, mais dans le cas de M'Pétiona, il semble qu'il aurait été préférable de mettre l'accent sur les organisations villageoises lors de la sensibilisation.

L'apport du comité de concertation, utile pour le projet dans le choix du site d'implantation de la $\mathrm{CEC}$, se révèle moins pertinent dans le choix des stratégies de sensibilisation. En effet, le processus de sensibilisation est plutôt lent et ne touche qu'une minorité de villageois. Cette stratégie permettra sans doute la mise en place de la CEC mais dans un processus plus lent qu'une stratégie qui aurait favorisé une collaboration active avec des organisations villageoises. Cette collaboration aurait permis d'accélérer le processus de sensibilisation et d'impliquer plus activement les divers groupes sociaux localisés.

57 Mais le type de projet proposé par la SDID, étant donné ses caractéristiques d'indépendance par rapport aux organisations locales, ne pouvait s'associer à celles-ci comme le font d'autres organisations d'épargne et de crédit en cours d'implantation dans la région. Ces dernières, en s'appuyant sur les $\mathrm{A} V$, encouragent la participation des membres de celles-ci, les chefs de famille. Par contre, dans ce cas, on peut se demander si les autres groupes sociaux, en particulier les femmes et les jeunes, participent aux activités de ces organisations.

Conclusion

58 L'analyse du changement social et du pouvoir local dans une perspective dynamiste et marxiste a permis d'éclairer le contexte social local, sans pour autant négliger les 
influences des classes dominantes et les particularités historiques du milieu, tous aspects qui expliquent la situation différenciée des groupes sociaux localisés face au projet de CEC.

59 Cette analyse indique l'impact social qu'un projet de développement doit être perçu en examinant les réactions des différents groupes sociaux au niveau interne tout en tenant compte des alliances qui se tissent et des intérêts en jeu dans les relations avec les groupes externes. L'approche adoptée a souligné l'écart existant entre, d'une part, les objectifs et les activités du projet de CEC et, d'autre part, la réalité du pouvoir local de M'Pétiona où différents groupes sociaux aux intérêts divers s'activent.

Dans mon questionnement, je présumais que ce projet constituerait un enjeu pour le pouvoir local et qu'il favoriserait certains groupes sociaux, en particulier ceux associés aux classes dominantes par leurs activités économiques, politiques et par leur orientation religieuse, c'est-à-dire les jeunes hommes et les adultes. J'ai souligné le contrôle des ânés sur les principales organisations locales, les transformations en cours à ce sujet et la marginalisation des femmes dans les pouvoirs villageois. J'ai aussi rappelé la spécificité de la CEC, c'est une coopérative indépendante des organisations économiques et politiques locales mais qui s'est associée à des organismes nationaux actifs dans le milieu pour s'implanter.

61 Mes constatations rejoignent celles plus générales de plusieurs auteurs (Billaz, 1981, Bouju, 1984, Guillermou, 1988, etc.) en ce qui concerne les transformations des pouvoirs villageois en Afrique de l'Ouest face aux interventions externes. On s'aperçoit qu'à M'Pétiona, le pouvoir traditionnel des aînés, toujours présent, est affaibli par les interventions externes. Le pouvoir des jeunes et des adultes est associé aux organisations externes et à l'islamisation croissante. Les femmes ont peu de contact avec les principales organisations externes et peu de pouvoir.

62 L'analyse de l'implantation du projet confirme en partie mon hypothèse. II faut toutefois nuancer la conclusion. En effet, dans ce cas, seuls les jeunes hommes sont associés à la CEC.

63 La plupart des adultes détenant un pouvoir localement, parce qu'ils sont membres de l'Association Villageoise (AV) et des conseils villageois, attendent de mieux connaître cette nouvelle organisation avant de l'appuyer.

64 En ce qui concerne les aînés, nous avons mentionné qu'ils appuient le projet mais de manière passive. Ils donnent leur consentement et mettent le projet entre les mains des jeunes.

65 Pour ce qui est des femmes, et malgré les faiblesses relevées dans les activités de sensibilisation du projet et la résistance des hommes, trois groupes ont tout de même déposé des provisions en vue d'adhérer à la Caisse.

Nos constatations suggèrent plusieurs questions qui trouveront leurs réponses dans l'évolution des rapports entre les divers groupes sociaux localisés villageois et les organismes externes. Ainsi, on peut penser que la collaboration active des jeunes avec le projet et les organismes externes favorisera l'émergence d'une nouvelle forme de pouvoir et des transformations dans l'autorité traditionnelle des aînés. Comment ces transformations seront-elles acceptées par le pouvoir local? Dans ce contexte, les femmes auront-elles la possibilité de s'impliquer plus intensément dans ce projet étant donné les contraintes sociales? Cela dépendra sans doute de l'intérêt manifesté pour la CEC par les différents groupes et des stratégies de sensibilisation du projet. 
67 de l'implantation du projet de CEC souligne l'importance d'une analyse du champ politique local prenant en considération les différentes instances de la réalité sociale. Nous avons montré la pertinence des variables telles que les catégories d'âge et de sexe, l'appartenance religieuse et la prise en considération du pouvoir endogène et du pouvoir exogène (les rapports entre la classe dominante et la paysannerie) pour appréhender les communautés rurales de cette région. L'appartenance ethnique ou clanique ne joue pas un rôle significatif dans notre cas. Les deux groupes ethniques et les forgerons, compte tenu de leur importance démographique, sont représentés dans les organisations villageoises. Cependant, c'est une variable à considérer dans d'autres contextes où il existe une différenciation sur cette base entre des groupes comme l'a mentionné Guillermou (1988). Tous ces éléments permettent d'accéder à une compréhension globale du changement social en cours dans un milieu spécifique.

\section{BIBLIOGRAPHIE}

Amselle J.-L. et M'Bokolo E. (Ed.), 1985, Au cœur de l'ethnie, Paris, Ed. La Découverte.

Amselle J.-L., 1985, "Socialisme et pré-capitalisme au Mali (1960-1982)", in Contradictions of Accumulation in Africa, New York, Sage publication, pp. 249-266.

Balandier G., 1984, Anthropologie politique (1967), Paris, P.U.F.

Balandier G., 1971, Sens et puissance, Paris, P.U.F.

Bariteau C. et al., 1983, Le pouvoir local. Documents de recherche $\mathrm{n}^{\circ} 1$. Laboratoire de Recherches Anthropologiques, Faculté des Sciences Sociales, Département d'Anthropologie, Université de Laval.

Billaz R. et Y. Diawara, 1981, Enquête en milieu rural sahélien, Paris, P.U.F.

Bouju J., 1984, Graine de l'homme, enfant du mil, Paris, Société d'Ethnographie.

Copans J. (Ed.), 1975, Sécheresses et famines au Sahel, Paris, Maspéro.

Dumouchel S. et Thede N., 1986, Femmes, épargne et crédit au Sahel, Montréal, Projet Solidarité Canada Sahel.

Guillermou Y., 1988, "Procès de production et formes de surtravail dans les sociétés rurales africaines", in Cahier des sciences humaines, vol. 24, 8, pp. 471-485.

Haubert T.M. (Ed.), 1985, Politiques alimentaires et structures sociales en Afrique, Paris, P.U.F.

Jonckers D., 1987, La société minyanka du Mali. Traditions communautaires et développement cotonnier, Paris, l'Harmattan.

Labatut J.-M., 1989, "La méthode anthropologique et la petite production marchande en Afrique", in Anthropologie et sociétés, vol. 13,3, pp. 73-98.

Ledrut R. (Ed.), 1979, Le pouvoir local, Paris, Anthropos.

Meillassoux Cl., 1977, Terrains et théories, Paris, Anthropos. 
Société de Développement International Desjardins (SDID), 1989, Projet pilote de développement de coopératives d'épargne et de crédit au Mali, Québec, Lévis, SDID.

Société de Développement International Desjardins (SDID), 1990, Études socio-économiques pour le projet pilote de, développement de coopératives d'épargne et de crédit au Mali, Québec, Lévis, SDID.

Terray E., 1969, Le marxisme devant les sociétés "primitives", Paris, Maspéro.

Vergès P., 1983, "Les classes sociales dans l'analyse localisée", in Sociologie du travail, vol. 2-83, pp. 226-232. 